\title{
BMJ Open Quality Using Lean Six Sigma methodologies to reduce risk of warfarin medication omission at hospital discharge
}

\author{
Ashlie Kallal, ${ }^{1}$ David Griffen, ${ }^{2}$ Cassie Jaeger (ib ${ }^{3}$
}

To cite: Kallal A, Griffen $D$, Jaeger C. Using Lean Six Sigma methodologies to reduce risk of warfarin medication omission at hospital discharge. BMJ Open Quality 2020;9:e000715. doi:10.1136/ bmjoq-2019-000715

- Additional material is published online only. To view please visit the journal online (http://dx.doi.org/10.1136/ bmjoq-2019-000715).

Received 25 April 2019 Revised 6 April 2020 Accepted 4 May 2020
Check for updates

(C) Author(s) (or their employer(s)) 2020. Re-use permitted under CC BY-NC. No commercial re-use. See rights and permissions. Published by BMJ.

${ }^{1}$ Pharmacy, Memorial Medical Center, Springfield, Illinois,

United States

${ }^{2}$ Emergency Medicine, Southern Illinois University School of Medicine, Springfield, Illinois, United States

${ }^{3}$ Operations Improvement,

Memorial Health System,

Springfield, Illinois, United States

Correspondence to

Dr Cassie Jaeger;

Hawk.Cassie@mhsil.com

\section{ABSTRACT}

Unintended omission of warfarin, an anticoagulant used to prevent and treat thromboembolic events, can lead to serious medical complications. These complications include increased medical costs, hospitalisations and significant patient harm, including increased risk of thrombosis and mortality. Chart review of discharged patients at our institution revealed an average of one patient/month with warfarin omitted from the discharge plan despite intention to continue therapy. Lean Six Sigma methodology was used to improve the process. A system alert was implemented in the electronic health record to alert providers of patients who received warfarin during admission, the discharge medication reconciliation was complete, and there was no prescription for warfarin. Date and time of last warfarin dose and international normalised ratio were included in the alert. Providers had the option to return to the chart to update the discharge medication plan and add the warfarin prescription or to choose an appropriate over-ride reason. The number of patients discharged without an intended warfarin prescription following alert implementation was reduced from $10.5 \%$ $(4 / 38)$ to $0 \%(0 / 40)$ (two proportion test, $p=0.03)$. Alert tracking enhanced the ability to identify patients at risk for warfarin omissions. Process sustainability has been achieved by embedding system alerts in the electronic health record to trigger process steps.

\section{INTRODUCTION}

In an urban tertiary care 500 bed hospital in Illinois, USA, chart review of every new start warfarin patient managed with onetime warfarin doses between July and August 2016 revealed one patient/month discharged without warfarin, despite the intent to continue therapy.

Warfarin (Coumadin) is an anticoagulant indicated for the prophylaxis and treatment of thromboembolic events including deep venous thrombosis and pulmonary embolism, and reduces the risk of death and thromboembolic events including stroke and embolisation after myocardial infarction. ${ }^{1}$ Warfarin has a narrow therapeutic index, requires daily dosing, has multiple drug-drug and drug-food interactions, and requires close monitoring of prothrombin time/international normalised ratio (INR). Unintentional anticoagulant omission increases the risk of a thromboembolic event. $^{2-5}$

At our institution, prescribers are primarily responsible for appropriate warfarin management. Order sets and clinical decision support which includes dose range, allergy and drug-drug interaction checking are available to assist and guide the safe and effective use of warfarin. Clinical rules and alerts are used in the electronic health record (EHR). Although we do not have a formal pharmacy warfarin dosing service, pharmacy plays an important role in the safe use of warfarin. Pharmacists evaluate warfarin therapy for appropriate dosing, duplicate therapy, drugdrug interactions, drug-disease interactions and drug-lab interactions. Pharmacists may order a baseline INR for patients starting warfarin therapy when one is not already available. Pharmacists monitor INR results and notify physicians of subtherapeutic and supratherapeutic INRs. Pharmacists also provide warfarin education to patients and families starting warfarin as a new medication. All warfarin doses are administered at a defined standard time of 1800 . Nursing must review the INR result and document the INR prior to administering warfarin.

At our institution, new start warfarin patients are often managed with daily onetime warfarin orders based on INR results. Once the warfarin dose is administered, the one-time order is no longer active. If a subsequent warfarin order is not placed, there was no prompt or reminder to the provider at discharge to address and prescribe the new warfarin therapy. Of our new start warfarin patients, $37 \%$ had no order available to the provider at the point of discharge medication reconciliation.

Oral anticoagulants are commonly implicated in adverse drug events and result in high hospitalisation costs. ${ }^{6}$ Although frequently associated with overdose or toxicity, one anticoagulant associated risk includes accidental discontinuation of therapy. ${ }^{8}$ Coombes et al, 
audited discharge prescriptions and found that $15 \%$ (49/329) were unintentionally omitted. ${ }^{9}$ Duguid et al, reported that $12 \%$ of patients had at least one prescription error at discharge, including unintended omission of orders for warfarin..$^{10}$ Another study found that the highest number of medication errors associated with oral anticoagulants were drug omissions $(32.5 \%, 270 / 831)$ which were most often a result of medications not being ordered $(35.9 \%, 97 / 270)$, followed by orders not being administered $(31.5 \%, 85 / 270)$ and orders being processed incorrectly $(27.8 \%, 75 / 270)$. In this study, $88.5 \%$ (239/270) of dose omissions were patients on warfarin, likely due to the practice of daily one time orders being written based on INR results for the day. ${ }^{11}$

Strategies to prevent dose omissions include improving transitions of care, electronic reminders, policy and specialised pharmacy teams. Day et al, improved transitions of care for patients discharged on warfarin through an order in the EHR that prompted physicians to include important warfarin management components including indication, target INR, duration of therapy, provider and next INR. Use of the order significantly improved documentation of key warfarin components at discharge. ${ }^{12}$ Others have recommended standardising the process for intentional medication 'hold' orders, implementing an active reminder for daily prescribing and a pharmacy driven daily medication list that includes medications discontinued within the previous 48 hours. ${ }^{13}{ }^{14}$ Scott and White Memorial Hospital implemented a High Risk Medication Team of pharmacists to help prevent unintended omission of high-risk medications, including warfarin. The pharmacists reviewed a daily report generated by the computerised order-entry system that identified patients on high-risk medications and reconciled discharge medications to prevent or correct discrepancies, including unintended omissions. ${ }^{15}$ An example of a policy driven solution to help prevent unintended warfarin omissions is to require a specific order when a provider's intent is to discontinue or withhold a dose for high-risk drugs. If warfarin is prescribed during an inpatient's stay but a discontinue order is not present, the discrepancy might be identified at medication reconciliation. ${ }^{16}$

One of Joint Commission's National Patient Safety Goals is to "reduce the likelihood of patient harm associated with the use of anticoagulant therapy'. ${ }^{17}$ The goal of this project was to decrease risk of warfarin omissions at discharge to 0 patients/month aligning with our institution's mission to improve the health of the people and communities we serve and our strategic priority to reduce preventable patient harm.

\section{METHODS}

Inpatients started on warfarin therapy, managed with onetime warfarin orders and warfarin indicated at discharge met inclusion criteria. Patients receiving warfarin prior to admission were excluded because a process already existed to reconcile warfarin at discharge. Patients managed with ongoing warfarin orders, patients with no warfarin indication at discharge, and patients discharged on other anticoagulants were considered out of scope for this project and excluded. Baseline chart review was performed for 2 months on every new start warfarin patient managed with one-time warfarin orders. Process measures included number of alerts that were deferred/ total alert responses, number of alert responses that resulted in a warfarin prescription/total alert responses and number of alert responses that warfarin was not indicated/total alert responses. Outcome measures included number of patients with an intended warfarin prescription that was omitted at discharge.

Our hospital uses Lean Six Sigma methodology for continuous improvement, with nearly $50 \%$ of the workforce exposed to some level of Lean Six Sigma training. System leadership prioritised the initiative, keeping in line with the system's quality and safety improvement priorities. The multidisciplinary project team included representation from executive leadership including senior vice president (VP) and chief medical officer, VP of operations, supervisor of medication safety, director of clinical informatics, pharmacy informatics coordinator, physicians, clinical pharmacists and nursing. Stakeholders were identified at the beginning of the project and represented on the team. Other stakeholders providing input included patients and families. In accordance with Lean Six Sigma, team members and stakeholders were selected based on involvement with impacted processes and/or patients. Team members attended biweekly meetings to provide process and subject matter expertise.

Lean Six Sigma Define, Measure, Analyse, Improve, and Control methodology was used as the framework to guide the team through each of the key decision points in the project. Tools used included supplier, input, process, output, customer (SIPOC) analysis to identify high-level process steps and customers. Critical to Quality tree (online supplementary figure 1) was completed to determine process requirements based on voice of the customer. Process mapping organised the tasks completed from different disciplines into one overall process and revealed potential gaps that the multidisciplinary team could visualise. To further investigate potential contributing factors, cause mapping was used to determine what conditions had to be true for an error to occur. Through cause mapping, the team identified that when these warfarin prescription omissions occurred, the patient, family, pharmacy and nursing staff were unaware of the omission. Cause mapping also identified a process gap that new start warfarin therapy managed with one-time warfarin orders were not available at discharge reconciliation and lacked a system prompt to the provider. A fishbone diagram (online supplementary figure 2 ) identified potential contributing factors including warfarin education not provided to the patient/family, discharge plan not reviewed by pharmacist, warfarin discharge education not completed, no warfarin order available to reconcile if one-time order already charted and lack of provider 
prompt/reminder. Five why's analysis was used to further elaborate on potential root causes.

Critical factors for warfarin prescription omission at discharge included the management of new-start warfarin patients with one-time orders and the lack of a system prompt and forcing function in the EHR. Of new start warfarin patients, $37 \%$ had no warfarin order available at discharge medication reconciliation for the provider to address. This occurred if the one-time order had already been administered and a subsequent warfarin order was not already placed. There was no prompt or reminder to the provider when this occurred. Previous prompts were lost during the transition from paper to electronic medication reconciliation, and it was unknown to the team if the functionality existed in the current EHR.

Potential solutions were identified through team brainstorming, organised into categories using an affinity diagram, and compared using an impact effort matrix. Despite being high effort, the team decided to implement a system alert in the EHR to prevent warfarin prescription omission at discharge based on high impact potential. Evidence-based guidelines from the American College of Chest Physicians on warfarin dosing were considered to determine an appropriate process point for intervention. In a culture exposed to alert fatigue, expected alarm frequency, impact and potential patient benefits were considered. Failure modes and effects analysis was completed to identify potential failures and the effects of those failures. Identified failure modes included the provider ignoring the alert or not responding appropriately, the alert firing to a provider that isn't managing the warfarin therapy, no warfarin charted on the electronic medication administration record leading to no alert, warfarin documented on the discharge plan as a new home medication but no prescription provided, warfarin prescription generated outside of discharge medication reconciliation resulting in an inaccurate discharge plan, and a discharge plan printed prior to the addition of warfarin. Severity risk, occurrence and detection of the potential process failures were low enough to proceed with the intervention. Provider alert education was communicated at department meetings, electronically at the point of EHR login, and via email communication.

Two-proportion test was used to test statistical significance in the percent of patients with warfarin omitted at discharge before and after the intervention. Statistics were performed in Minitab V.17.2.1. A $p<0.05$ was considered significant. This submission has been reviewed by the local institutional review board. It was determined that this project was not research involving human subjects. Patients or the public were not involved in the design, or conduct, or reporting, or dissemination plans of our research.

A system alert was implemented that fires to providers at chart closing once discharge medication reconciliation has been completed. The alert identifies patients that received warfarin during admission but there is no warfarin prescription at discharge. Alert firing is based on the following conditional logic: (1) if a patient received warfarin during admission and (2) warfarin is not a home medication and (3) there is no prescription for warfarin and (4) discharge medication reconciliation is complete then (5) the alert will fire at chart close. Last dose and INR were included in the alert as additional information for providers (figure 1A). Providers have the option to override the alert with an appropriate reason or return to the chart to add the warfarin prescription and to update the discharge medication reconciliation. Appropriate

\section{Warfarin Discharge Alert}

A) Discern: Close Chart - TESTING, BRIANNE (2 of 2)
Darfarin at DC
Discharge Med Rec is complete and there is no discharge order
If continue warfarin.
If this patient should continue warfarin therapy. please return to
Discharge Med Rec and add a prescription for warfarin.
Last Dose: 1 mg October 05, 2017 13:00:00 CDT
Last INR: 0.1 August $30.201709: 01: 00$ CDT
Alert Action
O Return Chart
O Override
History

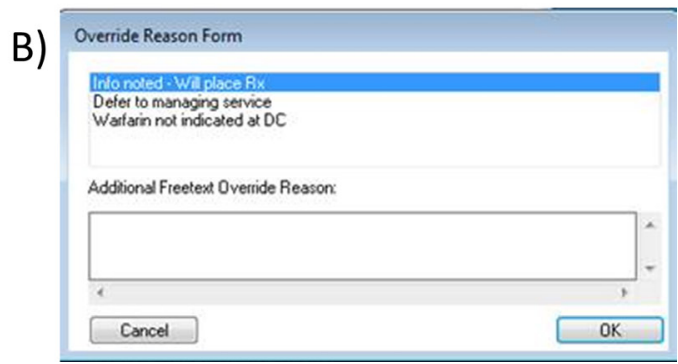

Figure 1 Screenshot of the warfarin discharge alert, which includes last dose and INR. Alert actions include return to chart or override (A). Providers that chose to override the alert are given the option to note that the prescription will be placed, defer to managing service or note that warfarin is not indicated (B). CDT, central daylight time; Info, information, INR, international normalised ratio; Med, medication; Rec, reconciliation. 


\section{Number of Patients Discharged without Intended Warfarin Prescription}

2.0

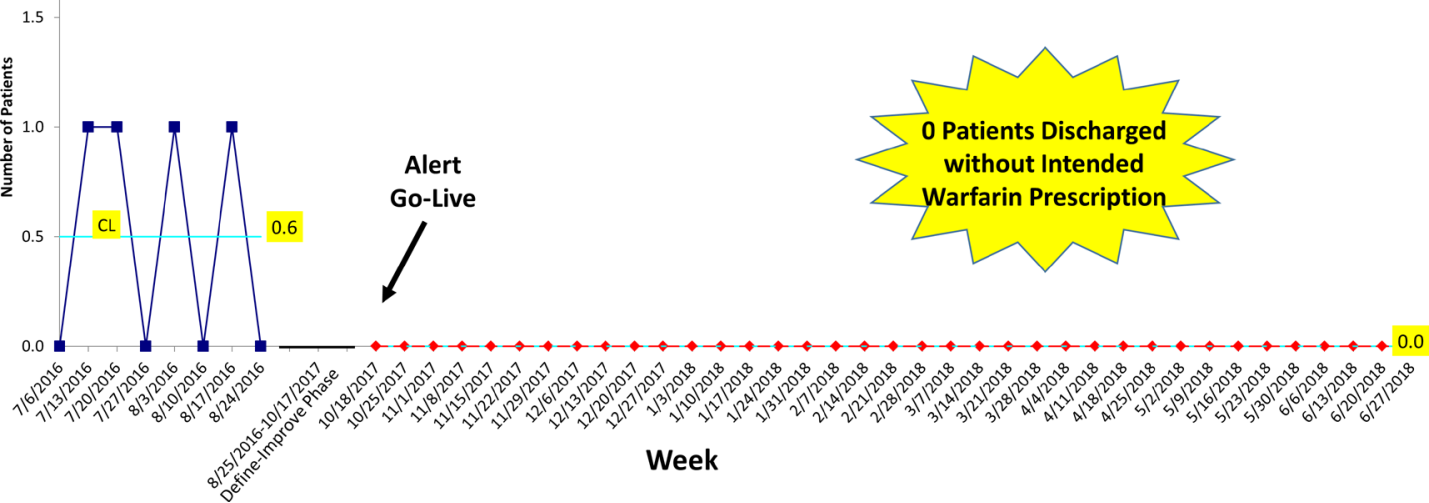

Figure 2 Omission of intended warfarin prescription at discharge was decreased from 1 omission/month to 0 omissions/month post-implementation of the system alert. (Two proportion test, $p=0.03$ ) the upper control limit (CL), shown as the red dotted line, represents $3 \mathrm{SD}$ from the mean. The solid blue centre line represents the average.

over-ride reasons include warfarin not indicated, will place prescription, and defer to managing service (figure 1B). The alert will continue to fire to any provider in the chart until the warfarin has been addressed.

The alert was built and tested by the information systems (IS) team and director of clinical informatics. The alert was activated in October 2017 following approval and provider education. Following alert implementation, a report was built to monitor the frequency of alert firing and provider alert response.

Our null hypothesis stated that implementing a system alert would not eliminate inadvertent warfarin prescription omissions at discharge. The alternative hypothesis stated that implementing a system alert would eliminate inadvertent warfarin prescription omissions at discharge.

\section{RESULTS}

Alert tracking reports enhanced the ability to identify patients at risk for warfarin omission and to evaluate provider alert response. Three months postimplementation, 96 alerts fired to 42 providers on 40 patients. The rate of intended warfarin prescriptions at discharge that were omitted decreased from 1 omission/ month to 0 omissions/month or $10.5 \%(4 / 38)$ to $0 \%$ $(0 / 40)$ (two proportion test, $\mathrm{p}=0.03$ ) (figure 2 ). Warfarin orders were subsequently placed following the alert on $30 \%(12 / 40)$ of patients, warfarin was not indicated at discharge in $60 \%(24 / 40)$ of patients, and the alert was deferred in $10 \%(4 / 40)$ of patients (online supplementary figure 3 ). A chart review was completed on all patients with the alert to ensure appropriateness and completion of the action. No patient safety events were reported related to warfarin prescription omission in the hospital's event reporting tool. Providers, staff and care coordination were impacted by work flow redesign to address the lack of forcing function in the EHR. The null hypothesis was rejected and the alternative hypothesis stating that a system alert would eliminate inadvertent warfarin prescription omissions at discharge was accepted.

\section{LESSONS AND LIMITATIONS}

When the alert was first implemented, 'warfarin not indicated at discharge' was the first of the three available over-ride reasons, followed by 'Info noted-will place prescription' and 'Defer to managing service'. When 'warfarin not indicated at discharge' is documented as the over-ride reason, subsequent alerts on the patient are suppressed. Based on initial data and feedback, the order of the alert over-ride reasons was changed and 'warfarin not indicated at discharge' was moved to the bottom of the list. This change was made to decrease the risk of harm if the first option was chosen in error and subsequent alerts were suppressed. Additionally, although the alert was tested by IS before implementation, it was identified after go-live that the alert was continuing to fire after discharge and adjustments were made.

A limitation of the project was that manual data collection was paused after initial confirmation that warfarin prescription omission at discharge was not an isolated event so that the Lean Six Sigma project team could work through the Define, Measure, Analyse and Improve phases. In addition, since chart review of all patients with the alert to ensure appropriateness of the provider response was manual, sustainability of this process step is a potential limitation. Introduction of new oral anticoagulants into the market and overall decreased trends in warfarin prescribing is a potential confounding factor that should be considered. ${ }^{18}$

Lean Six Sigma projects require a control plan to ensure results are sustained over time. Measures, target goals, data collection plans, review frequency and special cause response steps were defined. Measures include 
monthly provider alert responses, number of prescription omissions prevented, and $0 \%$ warfarin omission prescription rate monitored by alert data and chart audits (online supplementary figure 4). The control plan was communicated via the project team to promote sustainability and to further support the process owner. Process sustainability has been achieved by embedding a system alert and forcing function in the EHR. Provider-specific measures allow for individual feedback and retraining if needed. Ongoing evaluation through the control plan is enforced to ensure sustained improvements. Results and success of the project were shared with providers at the Pharmacy and Therapeutics Committee, Quality Safety Performance Committee and quarterly Lean Six Sigma meeting.

Processes are being spread to all affiliates in the health system via a Lean Six Sigma roll-out plan embedded in the control phase. Additional project extensions include application for similar medications with potential significant impact on patient safety. A limitation of this project is that interventions were limited to one hospital and results cannot be generalised.

\section{CONCLUSION}

Chart review indicated a rate of one patient/month discharged without warfarin prescription despite intent to continue. Following implementation of a system alert, which requires the provider to address warfarin at discharge, warfarin prescription omissions at hospital discharge were reduced to 0 . Reducing warfarin omissions aligns with the Joint Commission's National Patient Safety Goal to 'reduce the likelihood of patient harm associated with the use of anticoagulant therapy' and our institution's strategic priority to reduce preventable patient harm.

Acknowledgements Hamid Al-Johany, Edgard Cumpa, Laura Fiorito, Carol Nordstrom, Hilary Schneider, Heather Strawn, Veronica Walk, Charla Warren.

Contributors AK contributed to the study concept and design, acquisition of data, analysis of data, drafting of the manuscript, and study supervision. DG contributed to the drafting and critical revision of the manuscript. CJ contributed to analysis of data, drafting of the manuscript, and critical revision of the manuscript. All contributors reviewed the manuscript.

Funding The authors have not declared a specific grant for this research from any funding agency in the public, commercial or not-for-profit sectors.

Competing interests None declared.

Patient and public involvement Patients and/or the public were not involved in the design, or conduct, or reporting, or dissemination plans of this research.

Patient consent for publication Not required.
Ethics approval This submission has been reviewed by the local institutional review board. It was determined that this project was not research involving human subjects.

Provenance and peer review Not commissioned; externally peer reviewed.

Data availability statement № data are available.

Open access This is an open access article distributed in accordance with the Creative Commons Attribution Non Commercial (CC BY-NC 4.0) license, which permits others to distribute, remix, adapt, build upon this work non-commercially, and license their derivative works on different terms, provided the original work is properly cited, appropriate credit is given, any changes made indicated, and the use is non-commercial. See: http://creativecommons.org/licenses/by-nc/4.0/.

ORCID ID

Cassie Jaeger http://orcid.org/0000-0002-2726-6867

\section{REFERENCES}

1 Beckman MG, Hooper WC, Critchley SE, et al. Venous thromboembolism: a public health concern. Am J Prev Med 2010;38:S495-501.

2 Ageno W, Gallus AS, Wittkowsky A, et al. Oral anticoagulant therapy: antithrombotic therapy and prevention of thrombosis, 9th ED: American College of chest physicians evidence-based clinical practice guidelines. Chest 2012;141:e44S-88.

3 Bristol Myers Squibb. Coumadin (Warfarin)[package insert]. Princeton NJ: Bristol Myers Squibb, 2017.

4 Anthony M, Romero K, Malone DC, et al. Warfarin interactions with substances listed in drug information compendia and in the FDA-approved label for warfarin sodium. Clin Pharmacol Ther 2009;86:425-9.

5 Kirkwood TB. Calibration of reference thromboplastins and standardisation of the prothrombin time ratio. Thromb Haemost 1983;49:238-44.

6 Budnitz DS, Lovegrove MC, Shehab N, et al. Emergency hospitalizations for adverse drug events in older Americans. N Engl J Med 2011;365:2002-12.

7 Piazza G, Nguyen TN, Cios D, et al. Anticoagulation-associated adverse drug events. Am J Med 2011;124:1136-42.

8 Institute for Safe Medication Practices. High-alert medication feature: anticoagulant safety takes center stage in 2007. ISMP Med Saf Alert Acute Care 2007.

9 Coombes ID, Pillans PI, Storie WJ, et al. Quality of medication ordering at a large teaching hospital. Aust J Hosp Pharm 2001;31:102-6.

10 Duguid M, Gibson M, O'Doherty R. Review of discharge prescriptions by pharmacists integral to continuity of care.. J Pharm Pract Res 2002:94-5.

11 Andreica I, Grissinger M. Oral anticoagulants: a review of common errors and risk reduction strategies. Pennsylvania Patient Safety Advisory 2015:54-61.

12 Day M, Malone M, Burkeybile A, et al. Improving transitions of care for hospitalized patients on warfarin. Jt Comm J Qual Patient Saf 2016;42:425-31.

13 Pennsylvania patient safety reporting system. hold on to these orders. PA PSRS Patient Saf Advis 2006:33-4.

14 Cohen M. Preventing prescribing orders. In: Medication errors. 2nd edn. Washington, DC: American Pharmacists Association, 2007.

15 Martin ES, Overstreet RL, Jackson-Khalil LR, et al. Implementation of a specialized pharmacy team to monitor high-risk medications during discharge. Am J Health Syst Pharm 2013;70:18-21.

16 Kayser S. Crossed coverage. Agency for Healthcare Research and Quality:Cases and Commentaries 2007.

17 The Joint Commission. National patient safety goals effective January 2018: Hospital accreditation program. 2018.

18 Zhu J, Alexander GC, Nazarian S, et al. Trends and variation in oral anticoagulant choice in patients with atrial fibrillation, 2010-2017. Pharmacotherapy 2018;38:907-20. 